\title{
A NEW MOBILITY FORMULA FOR SPATIAL MECHANISMS
}

\author{
Charles Wampler \\ General Motors R\&D Center \\ 30500 Mound Road, MC 480-106-359 \\ Warren, Michigan 48090 \\ Email: charles.w.wampler@gm.com
}

\author{
Blake T. Larson \\ Arthur G. Erdman \\ Department of Mechanical Engineering \\ University of Minnesota \\ Minneapolis, Minnesota 55455 \\ Email: LarsonB1@asme.org; agerdman@me.umn.edu
}

\begin{abstract}
A new formula for predicting the mobility of spatial mechanisms is introduced. Instead of counting rigid links and the constraints between them, as is done in the usual Grübler-Kutzbach formulae, we count vertices and edges in a polyhedral model of the mechanism. It is well known that the conventional formula underpredicts the mobility of certain exceptional classes of mechanisms, and in particular, does not easily accommodate compound spatial mechanisms that contain planar or spherical sub-mechanisms. The new approach provides a correct mobility whenever the conventional formula does and accounts for planar and spherical sub-mechanisms in a simple manner. Additionally, we present simple modifications to correctly model certain mechanisms that have remote spherical centers. We illustrate the method on compound mechanisms constructed from scissors linkages.
\end{abstract}

\section{INTRODUCTION}

A necessary step in synthesizing a new mechanism or analyzing a given system is to determine the degrees of freedom of the device. At the outset of designing a mechanism or robot for a given task or class of tasks, the number of degrees of freedom of the desired device is specified, and one wishes to explore various kinematic topologies to achieve the desired functionality. One may also wish to obtain a mechanism with some special subset of the space of rigid motions, such as only translational degrees of freedom. In this "type synthesis" phase, it is conve- nient to employ a simple formula, based on a simple accounting of the number of links and the joint types connecting them, to predict the mobility of various linkage topologies under consideration. The mobility prediction is equal to the actual degrees of freedom when the link geometries are as general as possible within the assumptions of the accounting method. However, the degrees of freedom can exceed the mobility index when the links meet special conditions. The purpose of this paper is to introduce a new mobility formulation that, compared to existing approaches, more easily captures certain special configurations of links, thereby providing a tight mobility count for such mechanisms. In many cases, more modern refinements of the classical Grübler-Kutzbach formulae, especially those based on intersection and union of subgroups of the rigid motions, achieve the same goal. The approach presented here offers an alternative that seems simpler and more intuitive in many cases. We find it particularly apt for spatial mechanisms that have multiple centers where rotational joints intersect and for compound mechanisms built from scissors linkages.

The most familiar expressions for the mobility $M$ of a spatial mechanism of $N$ rigid links with $J$ joints are the GrüblerKutzbach formulae

$$
M=6 N-6-\sum_{j=1}^{J} c_{j}=\sum_{j=1}^{J} f_{j}-6 L, \quad \text { (spatial case) }
$$

where $c_{j}$ is the number of constraints imposed by joint $j, f_{j}$ is the number of freedoms allowed by joint $j$, and $L$ is the num- 
ber of loop closures [1,2]. Note that $c_{j}+f_{j}=6$. While these relations are usually attributed to Grübler and Kutzbach, many others have contributed to these and offered alternative formulations as well [3]. The justification for the first of these formulae begins with the observation that an unconstrained rigid body in three-space has 6 degrees of freedom, namely, three translations and three rotations. Thus, we begin with $6 N$ freedoms, subtract the total number of constraints imposed by the joints between the links, and subtract 6 to declare one of the links as the ground link. (That is, mobility counts the internal degrees of freedom, ignoring the rigid body motion of the mechanism as a whole). The second version of the formula recognizes that if we cut $L$ links to turn the mechanism into an open-tree topology, the resulting mechanism has mobility equal to the sum of the freedoms of its joints. It costs 6 degrees of freedom to reconnect each cut link to recover the original mechanism.

It is well-known that the spatial Grübler-Kutzbach formulae are incorrect when the joints are in special arrangements. In particular, if the mechanism is spherical, that is, if it has only rotational joints and these all intersect in a common point, then translations can be ignored, and the correct mobility is

$$
M=3 N-3-2 J=J-3 L, \quad(\text { spherical or planar })
$$

This formula uses the fact that each rotational joint has one degree of freedom, or, considering that only orientation is at issue, exerts two constraints. Translation can be ignored in the mobility count by placing the world origin and the origin of coordinates for each link all at the common intersection point of the joints. With these choices, the translation of every link is identically zero. The same formula applies to planar mechanisms, as these can be seen to be spherical mechanisms with the common point at infinity. (Alternatively, one may argue that motion in the plane consists of two translations and one rotation and thereby arrive at the same formula.) It is not widely recognized, but Eq. 2 actually holds on a somewhat broader class of mechanisms: instead of requiring all joints to intersect in a common point, it is sufficient that all joints meet in a common point for each loop closure. For example, one may use an output angle of one spherical four-bar as the input angle to another, and even though the two sub-mechanisms have different spherical centers, Eq. 2 predicts the correct mobility.

In addition to spherical and planar mechanisms, there exist other special arrangements of links. Herve [4] classifies mechanisms into three categories: "trivial," for which the GrüblerKutzbach formulae suffice; "exceptional," which may have some combination of intersecting, parallel, and perpendicular joint axes; and "paradoxical," in which more complicated relationships in the link lengths, twists, and offsets affect the mobility. Examples of the latter include the Bennett four-bar [5] or architecturally singular Stewart-Gough platforms [6], whose de- grees of freedom are higher than the mobility predicted by Eqs. 1 or 2. Paradoxical linkages do not submit easily to simple mobility accounting rules as their unexpectedly high degrees of freedom occur because all the links jointly satisfy one or more extra compatibility conditions. Without a full classification of all such exceptions, one can only conclude is that the degrees of freedom of a specific mechanism is greater than or equal to the mobility of the class of mechanisms to which it belongs. (See Eq. 3 below.) Even this rule has a possible exception, which we discuss below.

One general way to handle "overconstrained" linkages is to form kinematic closure equations and determine the dimension of their solution set. As the closure equations for many joint types, including revolute, prismatic, cylindrical, spherical, and planar, can be expressed as polynomials, numerical algebraic geometry can be applied to determine the degrees of freedom and to analyze the solution set $[7,8]$. However, such a heavy computational procedure is not convenient for the kind of experimentation one might wish to perform during type synthesis. If one is given the mechanism in a general configuration, then the co-rank of the Jacobian matrix of the closure equations gives the infinitesimal degrees of freedom of the mechanism. For examples using this approach, see $[9,10]$. This can be very useful, but it must be kept in mind that there may be fewer finite degrees of freedom than infinitesimal ones. Also, in linkages having a high degree of symmetry, it is common that one only knows how to assemble the mechanism in a symmetric configuration, which may not be a general one. For example, it may lie at the intersection of several solution components and therefore have extra infinitesimal degrees of freedom. By finding all solution components and their dimensions, numerical algebraic geometry overcomes this deficiency.

An alternative to a full analysis is to determine how to modify the Grübler-Kutzbach formulation to correctly account for some common exceptions, particularly, to handle compound mechanisms built by connecting planar, spherical, and spatial sub-mechanisms. Some work in this direction is presented in $[4,9,11,12]$

To be more precise about these matters, we must introduce some notations and definitions. Although we know of no exceptions to the following statements, they lack rigorous proofs, so we state them as conjectures. More careful statements of these are contained in [13]. Let $\mathbf{X}$ be the set of all mechanisms that have a given type. Here, type means an enumeration of the links, the types of joints between them, and any extra conditions imposed on the geometry of the links, such as intersecting joint axes. Let us assume that these conditions are complex analytic, so that $\mathbf{X}$ is an analytic subset of some complex Euclidean space. Let us assume also that all the loop closure equations are complex analytic, which is true for all joints derived from the lower-order pairs. (It is not necessarily true for cams and other higher-order joints.) Under these assumptions, the solution set of the closure equations, that is, the set of all assembly configurations of the 


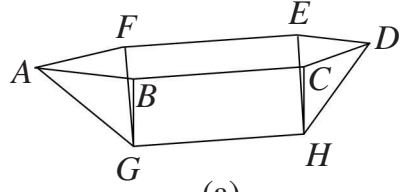

(a)

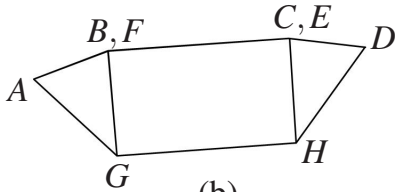

(b)
Figure 1. Mechanism that changes mobility

mechanism, is a complex analytic set. In practice, $\mathbf{X}$ is real and we are only interested in real assembly configurations, but it is advantageous to first consider the complex case and then restrict to the reals. A particular mechanism, $x \in \mathbf{X}$, may have several assembly modes and these are not necessarily of the same dimension. For an example, see [7], which shows that a seven-bar linkage built from Roberts cognate four-bars has both a coupler curve and up to six rigid assemblies. From this, we see that the mobility of a mechanism can be ambiguous; one should more properly speak of the mobility of a mechanism in an assembly mode. Even this can be slippery, for assembly modes of different dimension may meet, allowing the mechanism to move from one mobility condition to another. This is illustrated by the mechanism of Fig. 1 built from four triangles and two rigid rectangles. If $\triangle A G B=\triangle A G F$ and $\triangle D H C=\triangle D H E$, the configuration shown in Fig. 1a, which has mobility one, can be folded flat where its mobility increases to two as shown in Fig. $1 \mathrm{~b}$ since the rotations about $\overrightarrow{G B}=\overrightarrow{G F}$ and $\overrightarrow{H C}=\overrightarrow{H E}$ become independent.

For the purposes of this article, we will adopt the convention that $D O F(x)$ is the smallest dimension of any assembly mode of $x$. We make this choice because we wish to consider a mobility formula that does not require an assembly configuration to be given nor do we wish to carry out the calculations needed to find all the assembly modes given just the mechanism description $x$. Such a formula naturally leads us to the degrees of freedom of the lowest dimensional assembly mode as the other modes involve some special alignment of joint axes that is evident only given a configuration in that mode.

The real degrees of freedom of a mechanism is usually equal to its degrees of freedom in the complexes, but there are exceptions. Consider a planar four-bar in which the length of one link is equal to the sum of all the others. It is clear that this can be assembled in only one real configuration, with the three shorter links stretched to exactly span the long one. Over the complexes, the loop closure equations have a one-dimensional solution set: the real configuration occurs where two branches of this complex curve intersect. This is analogous to the solution set of $x^{2}+y^{2}=0$, which has only a single real point, $(x, y)=(0,0)$, even though the complex solution set consists of two lines, $x \pm y i=0$. Such points are always singular, and a mobility analysis based on the co-rank of the Jacobian matrix will indicate, at best, the complex dimension, not the smaller real dimension.
We must distinguish between two cases: either a general member of $\mathbf{X}$ can be assembled or not. In the first case, we define the mobility $\operatorname{Mob}(\mathbf{X})$, to be the largest integer $M$ such that the set $U=\{x \in \mathbf{X}: \operatorname{DOF}(x)=M\}$ is a dense, open subset of $\mathbf{X}$, that is, $\operatorname{Mob}(\mathbf{X})$ is the degrees of freedom of a general member of the type. In the case where a general member cannot be assembled, let $\mathbf{U}$ be the subset of $\mathbf{X}$ that can be assembled as a rigid structure: $\mathbf{U}=\{x \in \mathbf{X}: D O F(x)=0\}$. Then we define the mobility to be $\operatorname{Mob}(\mathbf{X})=\operatorname{dim} \mathbf{U}-\operatorname{dim} \mathbf{X}$, that is, the negative of the number of extra conditions which must be satisfied to assemble a mechanism of the given type.

From these definitions and the upper-semicontinuity of dimension, one may see that

$$
\operatorname{DOF}(x) \geq \operatorname{Mob}(\mathbf{X}), \quad \text { for all } x \in \mathbf{X} \text { (complex). }
$$

It is an immediate consequence that if $\mathbf{Y}$ is a subtype of $\mathbf{X}$, i.e., $\mathbf{Y} \subset \mathbf{X}$, then $\operatorname{Mob}(\mathbf{Y}) \geq \operatorname{Mob}(\mathbf{X})$. For this reason, a formula that correctly gives $\operatorname{Mob}(\mathbf{X})$ for, say, $\mathbf{X}$ equal to the class of spatial $6 \mathrm{R}$ loops may underpredict the mobility of some special types $\mathbf{Y}$ of $6 \mathrm{R}$ loops, such as the class of spherical 6R loops. We will say that a mobility formula $F(\mathbf{X})$ is tight for mechanism type $\mathbf{X}$ if $F(\mathbf{X})=\operatorname{Mob}(\mathbf{X})$. A formula that is tight for $\mathbf{X}$ may be loose for $\mathbf{Y} \subset \mathbf{X}$, meaning $F(\mathbf{Y})<\operatorname{Mob}(\mathbf{Y})$.

We emphasize that the preceding paragraph holds only for complex degrees of freedom. Letting $D O F_{\mathbb{R}}(x)$ be the degrees of freedom (dimension) of the smallest real solution component, we have

$$
\operatorname{DOF}_{\mathbb{R}}(x) \leq \operatorname{DOF}(x)
$$

Unfortunately, this leaves the comparison between $D O F_{\mathbb{R}}(x)$ and $\operatorname{Mob}(\mathbf{X})$ indeterminant in general.

We have the following two conjectures.

Conjecture 1: Equation 1 is tight for spatial mechanism types given only by a list of links and joints.

Conjecture 2: Equation 2 is tight for spherical and planar mechanism types given only by a list of links and joints. It is also tight for spatial mechanisms having only rotational joints in which each loop closure has a spherical center but the link parameters are subject to no additional relations.

As stated, these conjectures are not quite true, because we may have a mechanism that contains an overconstrained submechanism that cannot be assembled while also having sufficient extra degrees of freedom elsewhere to give a total nonnegative mobility. As it is not our purpose here to directly address these conjectures, we will not burden the exposition with the technicalities required to rule out such situations.

Our interest in stating these conjectures is to clarify the nature of exceptions to Eqs. 1 or 2, namely, any mechanism $x \in \mathbf{X}$ 


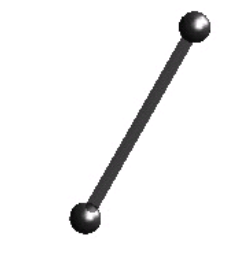

Figure 2. $V=2$

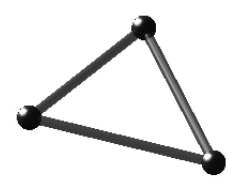

Figure 3. $V=3$

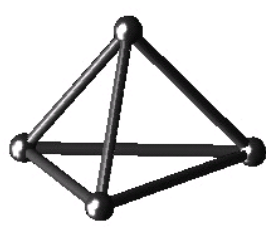

Figure 4. $\quad V=4$

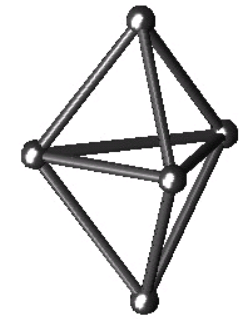

Figure 5. $\quad V=5$

\section{RIGID BODIES}

having $D O F(x)>\operatorname{Mob}(\mathbf{X})$ satisfies extra conditions not imposed simply by a list of links and their joints. For a mechanism type in which extra conditions are imposed, the mobility is defined as above, and we may seek a simple formula for it. We cannot expect any such mobility formula to give the correct degrees of freedom for all $x \in \mathbf{X}$, only for "general" mechanisms in $\mathbf{X}$, i.e., for a dense, open subset $\mathbf{U} \subset \mathbf{X}$. Thus, a more precise mobility formula than Eqs. 1 or 2 can only be obtained by more precisely describing the set $\mathbf{X}$ over which it applies. That is, the description of the mechanism type must include any special relations between the links and these relations must then be incorporated into the mobility calculation.

This paper presents mobility formulae that apply to classes of mechanisms that include both the fully spatial mechanisms where Eq. 1 applies and the spherical and planar mechanisms where Eq. 2 applies, as well as to many compound mechanisms having spherical or planar sub-mechanisms. Instead of focusing on rigid bodies, we focus on feature points of the links. The rigidity of the links is captured by constraining the distances between points of the same link and joints are modeled by allowing some points to belong to two or more links. Spherical and planar sub-mechanisms are properly accounted by selecting feature points that enforce these special relations. A mobility formula then follows by counting points and distance constraints.

The remainder of this paper is organized as follows. We first discuss the simplest case of polyhedral links connected by spherical and rotational joints. This gives a method of computing mobility correctly for spatial mechanisms that include spherical sub-mechanisms. The procedure is then generalized to treat joints having translational motion, including prismatic, cylindrical, and planar joint types. Next, we consider compound spatial mechanisms having planar sub-mechanisms and show how to correctly model the mobility that arises from certain special arrangements that are useful in constructing mechanisms that act as deformable surfaces. We end with several examples of mechanisms where the new approach has been useful in conceiving of compound mechanisms with interesting motions.

\section{MOBILITY OF CONNECTED POLYHEDRA}

For any mechanism built with lower-order pair joints, excluding helical joints, the salient features of each link are always points, lines, or planes. We will justify this statement later, but taking it as true for the moment, we see that a link may be naturally modeled as a polyhedron whose vertices, edges, and faces include the salient points, lines, and planes. A joint then constrains certain features of one polyhedron to lie on, or be equal to, certain features of another polyhedron. This leads to a mobility formula based on a simple count of vertices and edges. The simplest form accounts for spherical sub-mechanisms by insisting that one of the vertices be placed at the spherical center. A modified formula relaxes this condition by introducing an adjustment for remote spherical centers. As discussed in the next section, this modification presages a similar adjustment that arises when links represented by polyhedra are replaced by planar submechanisms having special properties.

\section{Rigid Bodies}

The defining characteristic of a rigid body is that the distance between any two points of the body is always preserved. If freely independent, each point would have three degrees of freedom, its $(x, y, z)$ coordinates. When the distance between two points is constrained, there is one fewer degree of freedom. That is, a simple rigid link in three-space consisting of just two feature points connected by an edge of constant length (see Fig.1a) has five degrees of freedom, since each point has three coordinates and the edge between them imposes one distance constraint: $2 \cdot 3-1=5$. In this manner, for the special case of $V=2$, we do not count the rotation around the line through the points that would be evident in a real link, which must have some thickness. If we wish to include that rotation in our count, an off-axis feature point must be introduced so that the link becomes a triangle. For $V \geq 3$, one may build up a free-floating rigid body with $V$ vertices by adding an appropriate set of $E=3 V-6$ edges such that its degrees of freedom is correctly counted as $3 V-E$. The arrangements for $V=2,3,4,5$ are illustrated in Figure 1. Notice that for five or more vertices, we do not include an edge between every pair of vertices, as the rigidity of the arrangement is already attained when $E=3 V-6$.

\section{Spherical and Rotational Joints}

A spherical joint, also known as a ball-and-socket joint, constrains the centers of the respective spherical surfaces of two 
links to be equal. In the representation of links as polyhedra, this simply means that two bodies share a common vertex. This is illustrated in Fig. 6. In Fig. 6a, two triangular-shaped links, labeled "A" and "B", are joined by a spherical joint at vertex $S$. Figure $6 \mathrm{~b}$ represents the polyhedral model, two triangles that share vertex $S$. A minimum of three vertices are required to represent each of these links; otherwise, one of the three rotational DOF of the spherical joint would be indeterminate. That is, if point 2 did not exist, then rotation about the line segment 1-S cannot be determined, because edges have only one dimension.

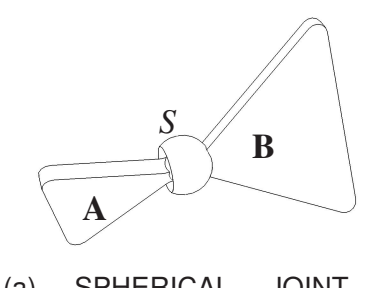

(a) SPHERICAL JOINT, TWO LINKS

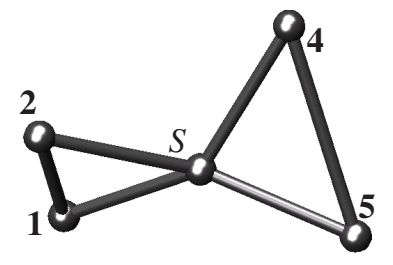

(b) POLYHEDRAL MODEL $(V=5, E=6, M=3)$

Figure 6. SPHERICAL JOINT BETWEEN TWO TRIANGULAR LINKS

A rotational joint is most simply represented in the polyhedral model as a pair of distinct points shared by two polyhedra. The rotation axis of the joint is the edge through the vertices. The location of the vertices is not unique, as any two points on the rotational axis can be chosen.

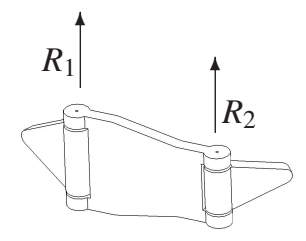

(a) 2R SPATIAL CHAIN

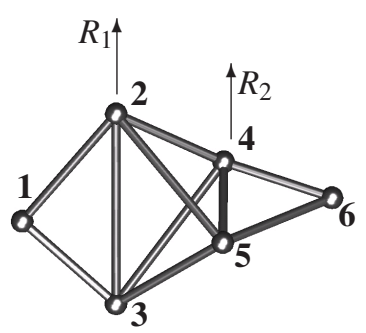

(b) POLYHEDRAL MODEL $(V=6, E=10, M=2)$
Figure 7. SPATIAL $2 R$ CHAIN

Figures 7 shows an example of an open $2 R$ chain. Figure $7 \mathrm{a}$ shows three links joined at two revolute joints; the general spatial case where joints $R_{1}$ and $R_{2}$ are skew. Figure $7 \mathrm{~b}$ shows a polyhedral model of that configuration, and because the rotation axes are skew the middle link is a tetrahedron. The edge $\mathbf{2 - 3}$ represents the $R_{1}$ axis, and edge $4-5$ represents the $R_{2}$ axis.

A spherical sub-mechanism occurs when several rotational joints meet in a common point. In Fig. 8 , joints $R_{1}$ and $R_{2}$ intersect at vertex $\mathbf{1}$, and the middle link becomes a triangle. To

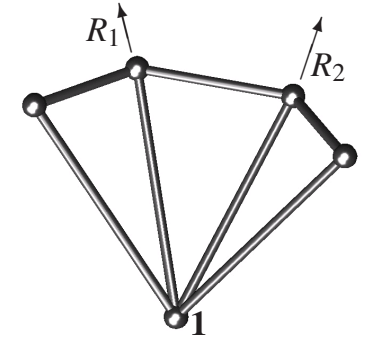

$V=5, E=7, M=2$

Figure 8. SPHERICAL 2R CHAIN

properly account for this, we simply select the common point as one of the two points representing each of the intersecting axes. For parallel joints, the common point is at infinity. For the purpose of mobility counting, it is convenient to draw such points as if they are finite, although other notations could be devised, see Fig. 16b. Figure 9 shows a closed-loop 4R spherical four-bar, where four triangles all share a single vertex, $\mathbf{5}$, with each other as well as an edge with each neighbor. Note that the four-sided loop formed by vertices 1-2-3-4-1 is not a rigid body. An additional edge between opposing vertices, either 1-3 or $\mathbf{2 - 4}$, would reduce the mobility by 1 , forming a rigid structure.

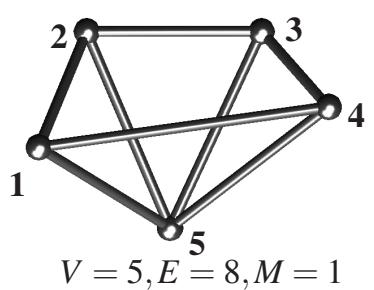

Figure 9. SPHERICAL FOUR-BAR POLYHEDRAL MODEL

\section{A Mobility Formula}

Using the ideas of the previous subsections, one can model any mechanism having only spherical and rotational links as a collection of vertices and edges. Accordingly, the mobility of the mechanism is given as

$$
M=3 V-E-6
$$

As in the first relations of Eqs. 1 and 2, we subtract 6 to fix one link and count only internal degrees of freedom. Using this formula for the mechanisms of Figs. 6, 7, 8, and 9 gives the correct mobility in all cases. Unlike Eqs. 1 and 2, Eq. 5 holds for both spatial and spherical mechanisms. 
Furthermore, Eq. 5 holds for mechanisms where Eq. 1 is not tight and Eq. 2 does not apply. Such mechanisms mix spherical sub-mechanisms with spherical joints. An example is shown in Fig. 10, having six triangles in a spherical 6R loop defined by vertices 1 through 7 . There is also a coupling between two opposing triangle faces 2-3-7 and 5-6-7 via two triangles 2-3-8 and 5-6-8 joined at spherical joint 8 . The two loops in this mechanism, 1-2-8-6-1 and 3-4-5-8-3, each have an independent degree of freedom. For this mechanism, Eq. 5 gives the correct result, $M=2$, while in contrast, Eq. 1 gives $M=-1$.

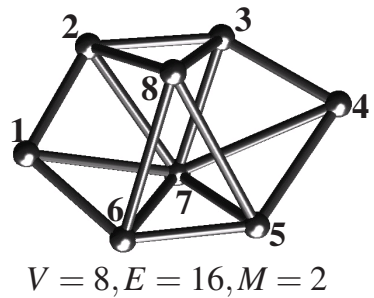

Figure 10. SPATIAL EIGHT-BAR WITH MOBILITY 2

\section{Joints with Translational Motion}

So far we have handled spherical and rotational joints, both of which only involve rotations. Among the lower-order pairs, prismatic, cylindrical, and planar joints all involve translation, independent of rotation. These can easily be incorporated into our formulation. Helical joints, which have rotation and translation coupled by the pitch of the screw, are not as easily accommodated, and will not be treated here.

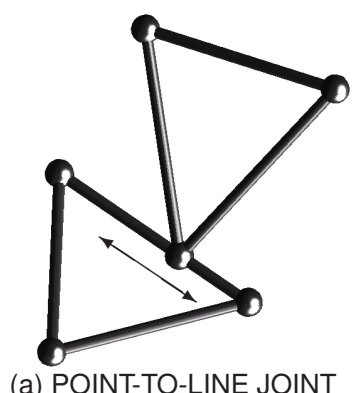

(a) POINT-TO-LINE JOINT

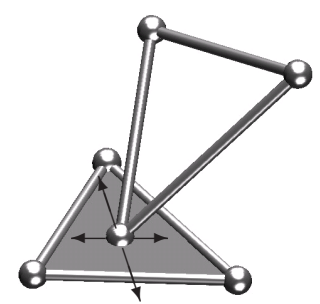

(b) POINT-TO-PLANE JOINT
Figure 11. BASIC LINEAR JOINTS

Polyhedra naturally contain linear elements: edges are line segments and faces are planar pieces. More generally, we may consider any two vertices of a polyhedron to define the line through them and similarly any three vertices define a plane. Accordingly, we may constrain a vertex of one polyhedron to lie on a line or plane of another polyhedron. Figure 11 illustrates each of these basic linear constraints. A point-to-line constraint costs two degrees of freedom (the point retains just one degree of freedom of motion along the line) whereas a point-to-plane constraint costs just one degree of freedom. Consequently, letting $L$ be the number of vertex-to-line constraints and $P$ be the number of point-to-plane constraints, the mobility formula becomes

$$
M=3 V-E-P-2 L-6
$$

One can combine several basic linear constraints to construct kinematic equivalents of the lower-order pairs. Constraining two vertices of polyhedron $A$ to lie on the same line of polyhedron $B$ is equivalent to a cylindrical joint between $A$ and $B$. Constraining three points of one to lie on a common plane of the other is equivalent to plane-plane contact between the polyhedra. Finally, a prismatic link may be modeled by constraining two vertices of $A$ to a line of $B$ and constraining a third point of $A$ to a plane of $B$ that contains the line. In this way, we generalize the polyhedral model to include rotational, prismatic, cylindrical, planar, and spherical joints.

\section{Adjustment for Remote Spherical Centers}

The correctness of Eqs. 5 and 6 in the presence of spherical sub-mechanisms depends on the choice of the sphere center as a vertex for each of the rotational joints around the loop, as was done in Figs. 8 and 9. Indeed, this is the only way a spherical submechanism can be specified using just vertices and edges without additional notation. However, suppose we begin with a spherical loop and mark two arbitrary points on each joint axis distinct from the spherical center. For example, the spherical four-bar of four triangles in Fig. 9 may be represented by the four tetrahedra in Fig. 12a. Without somehow noting that the joints intersect, we will obtain a lower mobility consistent with a spatial mechanism in which the joints do not intersect. Suppose then, that we simply note that the axes intersect in a remote center, as in Fig. 12a. To compute mobility correctly, we can start with an open-loop spherical four-bar as shown in Fig. 12b. To close this loop around the spherical center, it is merely sufficient to use a single pointto-line constraint, i.e. vertex 9 onto edge 1-5. The cost of this constraint, in terms of mobility, is -2 . This can be expressed as $\Delta M_{\text {true }}=-2$. But when the distances satisfy $a=a^{\prime}, b=b^{\prime}$, four vertices are reduced to two and two edges combine to one (by eliminating vertices $\mathbf{9}$ and 10). The apparent cost to close the loop is two vertices and one edge, i.e., $\Delta M_{\text {apparent }}=-5$. The common spherical center therefore adds $\Delta M_{\text {true }}-\Delta M_{\text {apparent }}=3$ freedoms to the system, which must be included in the formulation. To keep track of this, we mark the remote center as type $S_{+3}$, and letting $S_{+_{3}}$ denote the total number of such centers, the adjusted mobility formula becomes

$$
M=3 V+3 S_{+_{3}}-E-P-2 L-6
$$




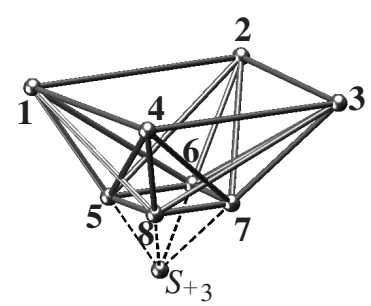

(a) CLOSED-LOOP SPHERI-

CAL FOUR-BAR

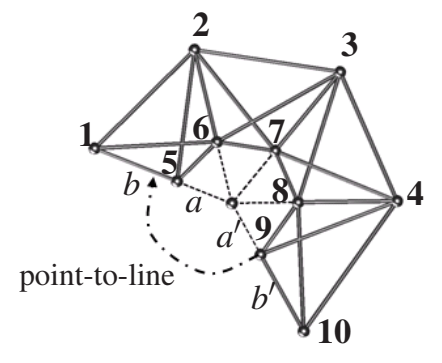

(a) OPEN-LOOP SPHERICAL FOUR-BAR
Figure 12. SPHERICAL LOOP WITH REMOTE CENTER

We could, of course, do without remote centers and instead force a common vertex as in Fig. 9. However, the $S_{+3}$ terms are included as a convenience and in anticipation of additional adjustment factors needed for other types of mechanisms discussed below.

\section{MOBILITY OF CONNECTED SUB-MECHANISMS}

In the previous section, we considered mechanisms formed from polyhedral links connected by joints between points, lines, and planes. Now, suppose we join several such mechanisms together. One may simply count up the mobility of the whole mechanism using Eq. 7 unless some kind of extra relations exist between the sub-mechanisms requires an adjustment similar to the remote center adjustment discussed previously. In this section, we consider one such class of mechanism that has a particular implementation using planar scissors sub-mechanisms.

Consider a one-degree-of-freedom mechanism that has four output vertices, illustrated schematically in Fig. 13a. Assume that the motion of the mechanism is such that the four vertices are always coplanar. Therefore the lines through any two pairs of vertices, say line $A B$ and line $C D$, intersect. Further, assume there are several such mechanisms connected sequentially as in Fig. 13b whose motion characteristics are such that all the indicated lines intersect in a common point. Then, we know to check the possibility that closing a loop around this common point to form the mechanism of Fig. 13c might, as in the case of remote spherical centers, produce a disparity between the true number of constraints required to close the loop and the apparent number implied by counting vertices. Indeed, if the whole chain of submechanisms is constructed such that the output distances equal the input distances, i.e., if $a=a^{\prime}, b=b^{\prime}$, then the true cost of closing the loop is $\Delta M_{\text {true }}-2$, whereas the apparent cost is two vertices, i.e., $\Delta M_{\text {apparent }}=-6$. Therefore, a correct mobility count requires an adjustment of +4 . Accordingly, we mark the remote center in this case as type $S_{+_{4}}$.

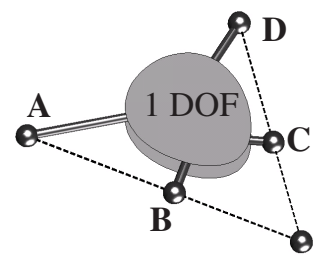

(a) PLANAR SUBMECHANISM

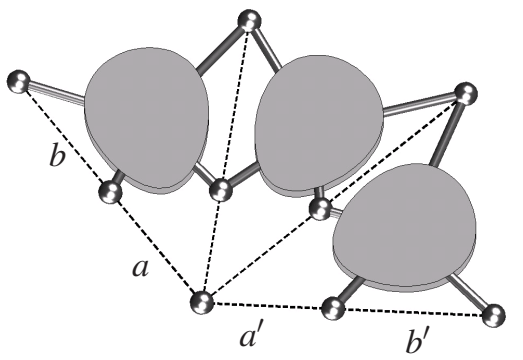

(b) OPEN CHAIN OF THREE PLANAR SUBMECHANISMS

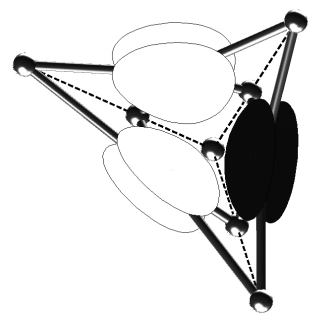

(c) CLOSED CHAIN OF THREE PLANAR SUBMECHANISMS

Figure 13. SUBMECHANISMS HAVING A REMOTE CENTER

The situation just described can arise in many ways. One is that each of the sub-mechanisms in the series is an identical symmetric scissors linkage (see Fig. 14a). If the number of submechanisms is even the conditions are met by identical asymmetrical scissors attached in mirror-image pairs (see Fig. 14b).

A related type of closure is illustrated in Fig. 15, where a series of sub-mechanisms are connected all sharing a vertex at a common center. For this configuration, if the design of the submechanisms is such that $a=a^{\prime}$, the true cost of closing the loop is again $\Delta M_{\text {true }}=-2$, whereas the apparent cost is one vertex, i.e., $\Delta M_{\text {apparent }}=-3$, hence the closure requires an adjustment of +1 . This may be indicated on a diagram of the mechanism as a vertex of type $S_{+_{1}}$. Similar to above, the condition for a $S_{+_{1}}$ vertex is met by an even number of scissors linkages connected in mirror-image pairs.

We may account for all such special arrangements by letting $S_{+}$denote the total number of vertices of that type. Accordingly, 


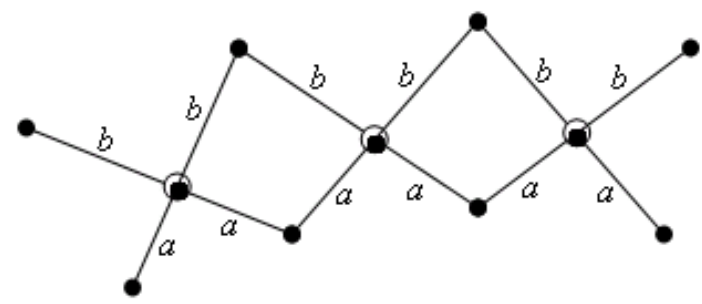

(a) CHAIN OF THREE SYMMETRIC SCISSORS

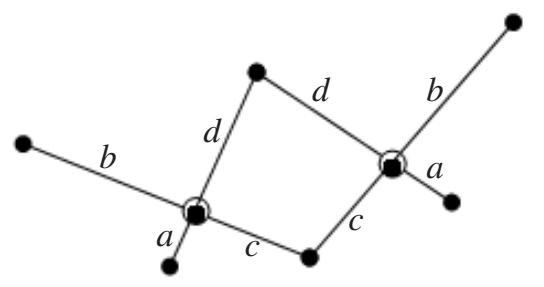

(b) FLIPPED PAIR OF ASYMMETRIC SCISSORS

Figure 14. SCISSOR SUBMECHANISMS

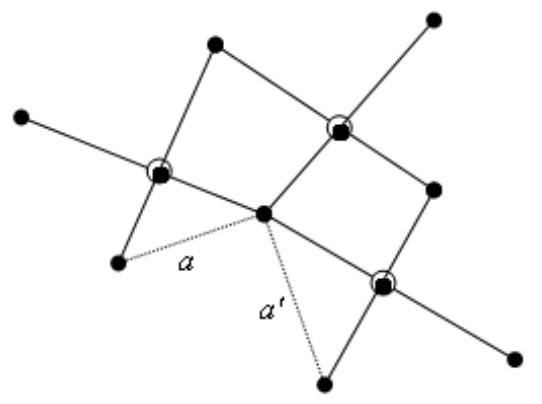

Figure 15. THREE SCISSORS WITH A COMMON CENTER

the mobility formula becomes

$$
M=3 V+\sum_{k} k S_{+_{k}}-E-P-2 L-6
$$

This count includes the internal vertices, edges, etc., of the submechanisms. Instead, one can summarize the internal constraints of each sub-mechanism for the purpose of counting the mobility of the whole. For example, a scissors mechanism has four exposed vertices which contribute 12 to the mobility, but its true contribution is its internal mobility of 1 plus 6 degrees of freedom of rigid-body motion, i.e., 7 total. Accordingly, the internal constraints must add up to -5 . So, if we count $X$ scissors submechanisms and their exposed vertices, ignoring their internal vertices and edges, the mobility formula becomes

$$
M=3 V+\sum_{k} k S_{+_{k}}-E-P-2 L-5 X-6
$$

One may define similar shortcuts for other types of submechanisms.
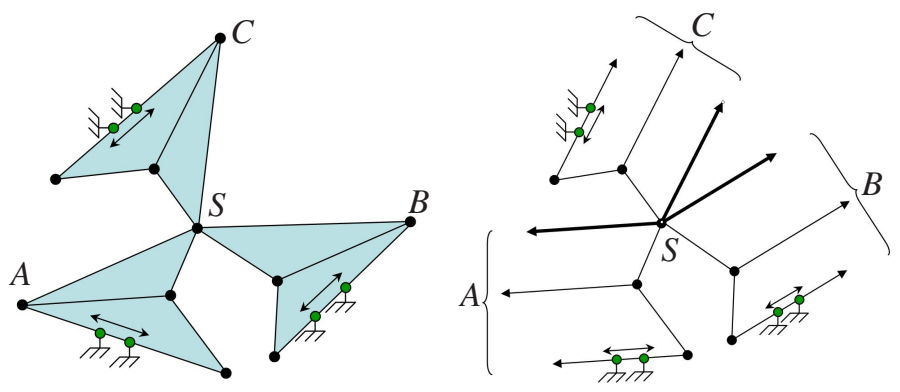

Figure 16. PARALLEL-LINK 3CRS MECHANISM

Remark: As a variant on all the preceding formulae, we may elect to declare one link the immobile ground link, omit any vertices of that link from the vertex count $V$ (and omit edges between any two of these), and drop the " -6 " from the end of the formula. Counting in this manner, our final formula becomes

$$
M=3 V+\sum_{k} k S_{+_{k}}-E-P-2 L-5 X \quad \text { (ignoring ground). }
$$

\section{EXAMPLES}

\section{A Translational Mechanism}

Consider the parallel-link mechanism shown in Fig. 16a, having three CRS legs meeting at a common spherical center $S$. Fixing the ground link, the mechanism has $V=10, E=15$, $L=6$, for which Eq. 10 gives mobility $M=3 \cdot 10-15-2 \cdot 6=3$. Letting vertices $A, B$, and $C$ move off to infinity, we have the mechanism of Fig. 16b having parallel axes. The mechanism still has three degrees of freedom, but now as point $S$ moves, the lines $\overrightarrow{S A}, \overrightarrow{S B}$, and $\overrightarrow{S C}$ never change direction. Hence, $S A B C$ forms a rigid frame, shown with bold lines in Fig. 16b, which translates in three degrees of freedom but does not rotate. A platform link can be built on this frame to obtain the translational parallel-link mechanism due to Tsai [14]. This mechanism is an exceptional linkage in the Herve classification system.

\section{Spherical Scissors Linkages}

Consider any single-loop or multiloop spherical mechanism built from identical unit links, each subtending the same angle, say $\alpha$, on the sphere. A rigid binary link may be just one unit link or it may be constructed by combining equilateral triangles, each with side length $\alpha$. Tertiary or higher links are also constructed from such triangles. Now modify the linkage by replacing each of the unit links with an identical symmetric scissors linkage, each rotational joint of the spherical linkage becoming a pair of spherical joints as in. For each independent loop of the original mechanism, we have a $S_{+4}$ remote center. Suppose the original spherical mechanism has $M$ degrees of freedom. The 
modified linkage will have $M+1$ degrees of freedom; the extra freedom is a simultaneous scissoring of all the unit links, changing $\alpha$ in unison. For example, consider a spherical fourbar. Before modification, it appears as in Fig. 9 with four identical isosceles triangles as sides. It has $V=5, E=8$, and thus $M=3 \cdot 5-8-6=1$. After modification, it has $V=8, S_{+_{4}}=1$, $X=4$, and thus $M=3 \cdot 8+4-5 \cdot 4-6=2$. Since the extra degree of freedom depends on the identical scissors elements, such linkages are paradoxical in the Herve classification.

If the symmetrical scissors have lengths $a=b$, where $a$ and $b$ are as shown in Fig. 14a, then the external vertices define parallel lines, $\alpha=0$, even as the scissors operate. The mobility is as before, but now the linkage is a planar one that grows and shrinks in the plane as the scissors act.

\section{Collapsible Polyhedra}

The Hoberman Sphere is a children's toy that performs a dramatic radial expansion from a spiny collapsed shape to a large spherical shape with a single degree of freedom. The key element of the Hoberman Sphere is a scissor pair designed such that the angle $\alpha$ defined by its exterior points $\mathbf{A}, \mathbf{B}, \mathbf{C}$, and $\mathbf{D}$ remains the same as shown in Fig. 17. Such an element may take several forms [15], and may even consist of multiple scissor pairs joined in series. The Hoberman-style elements may be connected by spherical joints as shown in Fig. 14 to form various collapsible shapes. We will use the polyhedral model to compute the mobility of a collapsible cube.

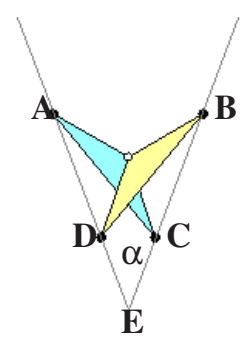

COLLAPSED ELEMENT Figure 17. HOBERMAN-STYLE SCISSOR ELEMENT

A cube is a regular polyhedron with 8 vertices, 12 edges, and 6 faces. To convert this into a Hoberman-style collapsible object, we replace each edge with a single DOF sub-mechanism, such as a scissor pair or chain of scissor pairs, that collapse to the same point. Figure 18a shows how a four-edged cube face can be replaced by four scissor pairs. The geometry of the scissor pair limits the range of motion of the mechanism; we can replace each single scissor pair with multiply-connected scissor pairs joined by revolute joints as long as the external vertices remain coplanar, maintain the same angle, and maintain equal input and output distances, that is, $|A E|=|B E|$ and $|D E|=|C E|$.

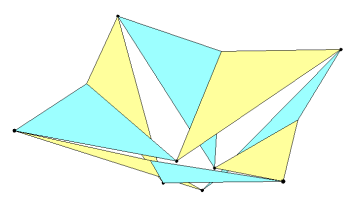

(a) FOUR CUBE EDGES REPLACED BY SCISSOR PAIRS

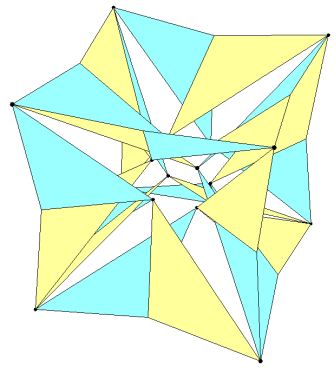

(b) ASSEMBLED COLLAPSIBLE CUBE

Figure 18. COLLAPSIBLE CUBE

Just as three edges meet at each cube vertex, three submechanisms are joined at a "corner" of a collapsible cube (see Fig. 18b). The difference, however, is that the sub-mechanisms meet at two vertices for each corner. The joints at these corners are all spherical. Each cube face translates to a closed loop of sub-mechanisms. In total, there are twelve scissor pairs, each of which could be replaced by a series of scissors. The scissor-pairs are constructed such that the central angle $\alpha$ matches the angle subtended from the center of a cube by an edge of the cube, that is $\alpha=\cos ^{-1}(1 / 3)$.

To count the mobility of the collapsible cube, first lock all the scissor pairs such that $|A E|=d$ for some common distance $d$. Each scissor pair is then equivalent to an identical isosceles triangle with sides $d$ and apex angle $\alpha$. These fit together as a set of 9 vertices, the 8 vertices of a cube and its center, joined by 20 edges, being the 12 edges of a cube plus 8 edges from the center to each outer vertex. The mobility of this arrangement is, by Eq. $5, M=3 \cdot 9-20-6=1$. It can be verified that this is the correct mobility for a general set of nine vertices connected by twenty edges in the topological pattern of the cube. However, recall from Eq. 4 that for special mechanisms, the number of real degrees of freedom can decrease. This indeed happens for the perfect cube arrangement. Each diagonal of the cube is exactly spanned by two edges connected at the center. Similar to the example of the degenerate four-bar with three short links exactly spanning the fourth longer link, the perfect cube is a isolated real point: the mechanism has $D O F_{\mathbb{R}}=0$ for fixed $d$. When the scissor pairs are unlocked, the entire collapsible cube 
is seen to have mobility $D O F_{\mathbb{R}}=1$. It is essential that the scissor pairs be identical so that as $|A E|=d$ changes, the secondary distance $|D E|$ also matches on all the scissor pairs. We see that the whole mechanism has just one finite real degree of freedom: it expands and contracts. However, the mechanism is singular at every position, so in addition to the finite motion, it has an extra infinitesimal motion. Any physical instantiation of it will tend to flex easily in this extra mode. This mechanism could be said to be doubly paradoxical, as it gains an extra degree of freedom in complex space due to the special geometry of the Hoberman scissors (hence is paradoxical according to Herve), yet in real space it loses a degree of freedom as a finite motion in the complexes degrades to an infinitesimal one in the reals.

A similar analysis can be applied to other collapsible shapes. For a regular tetrahedral shape, which has 4 vertices and 6 edges, replace each of the 6 edges with a scissors pair. Construct Hoberman-type scissor pairs matching the six outer edges and connect them. With the scissors locked, we have 5 vertices and 10 edges for a mobility of $M(\mathbf{X})=3 \cdot 5-10-6=-1$. This is the correct mobility for the mechanism type $\mathbf{X}$ consisting of six triangles with incompatible central angles; the links cannot generally be assembled in the tetrahedral pattern. However, since we have chosen a particular instance, $x$, having all central angles equal and compatible with a regular tetrahedron, they do assemble, and we have $D O F_{\mathbb{R}}(x)=\operatorname{DOF}(x)=0>\operatorname{Mob}(\mathbf{X})$. Hence, the locked-scissors state has mobility zero, while allowing them to unlock gives a mobility $M=1$. The collapsible tetrahedron is thus seen to expand and collapse only, always maintaining the shape of a regular tetrahedron.

\section{CONCLUSIONS}

We have presented alternatives to the traditional GrüblerKutzbach mobility formulae. In the case of just spherical and rotational joints, our new formula (Eqn 5) is based on a simple count of the vertices and edges in a polyhedral model of the assembled links. This single formula applies to equally well to spatial, spherical, and planar linkages. We also show how to adapt the formula to include prismatic, cylindrical, and planar joints, and to correctly count the mobility of certain compound spatial mechanisms formed by connecting special planar linkages. This last class of mechanisms includes some collapsible polyhedra built with scissors linkages.

We also clarify some subtleties relating to the mobility of mechanism families. The mobility of a sub-family may be larger than that of the family containing it, but considering dimensions in complex space, the mobility never decreases. The real mobility, that is, the dimension of the real solution set of the closure equations, usually is equal to the complex mobility, but in some instances it may decrease. We illustrate this with an example of a collapsible cube which both gains its collapsible degree of freedom due to special link geometry (Hoberman scissors) and loses a degree of freedom into a real singularity.

\section{REFERENCES}

[1] Grübler, M., 1917. Getriebelehre: eine Theorie des Zwanglaufes und der ebenen Mechanismen. Springer, Berlin.

[2] Kutzbach, K., 1929. "Mechanische leitungsverzweigung, ihre gesetze und anwendungen". Maschinenbau, der Betrieb, 8, pp. 710-716.

[3] Gogu, G., 2005. "Mobility of mechanisms: a critical review". Mechanism and Machine Theory, 40(9), pp. 10681097.

[4] Hervé, J. M. "Analyse structurelle des mecanismes par groupe des deplacements". Mech. Mach. Theory, 13.

[5] Bennett, G. T., 1903. "A new mechanism". Engineering, 76, pp. 777-778.

[6] Husty, M. L., and Karger, A., 2000. "Self-motions of griffis-duffy type parallel manipulators". In Proc. 2000 IEEE Int. Conf. Robotics and Automation, p. CDROM.

[7] Sommese, A. J., Verschelde, J., and Wampler, C. W., 2004. "Advances in polynomial continuation for solving problems in kinematics". Journal of Mechanical Design, 126(2), pp. 262-268.

[8] Sommese, A. J., and Wampler, C. W., 2005. The Numerical Solution of Systems of Polynomials Arising in Engineering and Science. World Scientific, Singapore.

[9] Rico, J. M., Gallardo, J., and Ravani, B., 2003. "Lie algebra and the mobility of kinematic chains". Journal of Robotics Systems, 20, pp. 477-499.

[10] Rico, J. M., and Ravani, B., 2007. "On calculating the degrees of freedom or mobility of overconstrained linkages: single-loop exceptional linkages". J. Mech. Design, 129(3), pp. 310-311.

[11] Zhao, J.-S., Feng, Z.-J., and Dong, J.-X., 2006. “Computation of the configuration degree of freedom of a spatial parallel mechanism by using reciprocal screw theory". Mechanism and Machine Theory, 41(12), 12, pp. 1486-1504.

[12] Hunt, K. H., 1990. Kinematic geometry of mechanisms. Oxford University Press, New York.

[13] Sommese, A. J., and Wampler, C. W., 2007. Mechanism spaces. in preparation.

[14] Kim, H. S., and Tsai, L.-W., 2002. "Evaluation of a cartesian parallel manipulator". In Advances in Robot Kinematics, Kluwer Academic Publishers, pp. 21-28.

[15] Patel, J., and Ananthasuresh, G. K., 2006. "A kinematic theory for planar Hoberman and other novel foldable mechanisms". In Proc. ASME 2006 Intl. Design Eng. Tech. Conf., ASME, pp. 1-9. 